\title{
MODAL ANALYSIS OF DAMAGED STRUCTURES BY THE MODIFIED FINITE ELEMENT METHOD
}

\author{
NGUYen CaO MENh, NGUYen TIEN KHIEM, \\ DaO NHU MaI, NGUYEN VIET KHOA \\ Institute of Mechanics, 224 Doican, Hanoi, Vietnam
}

\begin{abstract}
The classical 3D beam element has been modified and developed as a new finite element for vibration analysis of frame structures with flexible connections and cracked members. The mass and stiffness matrices of the modified elements are established basing on a new form of shape functions, which are obtained in investigating a beam with flexible supports and crack modeled through equivalent springs. These shape functions remain the cubic polynomial form and contain flexible connection (or crack) parameters. They do not change standard procedure of the finite element method (FEM). Therefore, the presented method is easy for engineers in application and allows to analyze eigen-parameters of structures as functions of the connection (or crack) parameters. The proposed approach has been applied to calculate natural frequencies and mode shape of typical frame structures in presented examples.
\end{abstract}

\section{Introduction}

The frame structures with semi-rigid (or flexible) connection have been studied in series of papers by Lui and Chen [2-4], Shi and Atluri [5], Kawashima and Fujimoto [6]. However, the methods proposed in their studies are limited either by the application for only some individual cases or by the difficulty for engineers in application. Chan and Ho [7] have suggested a new approach for calculating the mass and stiffness matrices of element basing on specific shape functions derived from solving the equilibrium equations of beam. The advantage of the method is keeping the cubic form (but with coefficients depending on connection parameters) of shape functions and the same as well known procedure of FEM. Some analytical aspects of the dynamic problem for beam-column with flexible supports have been investigated by Pedersen in [8].

Very few papers were contributed to develop the FEM for analysis of frame structures with cracked members. Most of researches in this field are limited to a description of crack nature rather investigating the influence of crack on the behaviour of structures especially dynamical, because of the complexity of the nature of crack. There is a simple approach [9-11, etc.], that regards to changes in the geometric properties of member due to crack. Most of them were completed 
by either classical FEM or analytical one and concerned only to simple structures such as beam column $[12,13]$.

The present study is addressed to the development of FEM in free vibration analysis of frame structures with flexible connections and cracked members. The basis of this investigation is the idea of using modified appropriate shape functions describing the-flexible-connection and crack of beam, which will be modeled below by equivalent springs. The spring parameters will be introduced so that their trivial (zero) values correspond to the classical model of beam. In this context, these parameters are called damage one and structure with such members is understood as damaged. Therefore undamaged structure is identical to the classical model of the structure.

The main subject of this study is to construct the shape functions depending on the damage parameters. Then the consistent mass and stiffness matrices will be calculated by formulas as well known in FEM. Subsequent procedure is followed the classical case in FEM.

Proposed approach is applied to calculate natural frequencies and modes of beam and frame structure in examples. Results obtained were compared with known one and give accepted agreement. As mentioned in [5], the suggested method is also efficient, relatively simple and can easily be accepted by engineers in practice.

\section{Beam Element - General Equation}

The beam finite element well known in FEM is described by 12 DOF called nodal displacements or generalized co-ordinates in the local system:

$$
U_{e}^{T}=\left(U_{1}^{e}, \ldots, U_{12}^{e}\right)=\left(u_{1}, v_{1}, w_{1}, \theta_{1}, w_{1}^{\prime}, v_{1}^{\prime}, u_{2}, v_{2}, \theta_{2}, w_{e}^{\prime}, v_{2}^{\prime}\right) .
$$

After the nodal displacements have been defined, it is important to express displacements $u(x), v(x), w(x), \theta(x)$ at section $x$ in term of the nodal ones:

$$
\begin{aligned}
u(x) & =\rho_{1}(x) u_{1}+\rho_{7}(x) u_{2}, \\
\theta(x) & =\rho_{4}(x) \theta_{1}+\rho_{10}(x) \theta_{2}, \\
v(x) & =\rho_{2}(x) v_{1}+\rho_{6}(x) v_{1}^{\prime}+\rho_{8}(x) v_{2}+\rho_{12}(x) v_{2}^{\prime}, \\
w(x) & =\rho_{3}(x) w_{1}+\rho_{5}(x) w_{1}^{\prime}+\rho_{9}(x) w_{2}+\rho_{11}(x) w_{2}^{\prime} .
\end{aligned}
$$

The selected so-called shape functions $\rho_{j}(x), j=1, \ldots, 12$, form the shape matrix

$$
H(x)=\left(\begin{array}{cccccccccccc}
\rho_{1} & 0 & 0 & 0 & 0 & 0 & \rho_{7} & 0 & 0 & 0 & 0 & 0 \\
0 & 0 & 0 & \rho_{4} & 0 & 0 & 0 & 0 & 0 & \rho_{10} & 0 & 0 \\
0 & \rho_{2} & 0 & 0 & 0 & \rho_{6} & 0 & \rho_{8} & 0 & 0 & 0 & \rho_{12} \\
0 & 0 & \rho_{3} & 0 & \rho_{5} & 0 & 0 & 0 & \rho_{9} & 0 & \rho_{11} & 0
\end{array}\right)
$$


In addition, following matrices are introduced

$$
[m]=\rho F I_{4 \times 4}, \quad[E]=\operatorname{diag}\left\{E F, G J_{x}, E J_{z}, E J_{y}\right\}
$$

and

$$
\bar{H}(x)=\left(\begin{array}{cccccccccccc}
\rho_{1}^{\prime} & 0 & 0 & 0 & 0 & 0 & \rho_{7}^{\prime} & 0 & 0 & 0 & 0 & 0 \\
0 & 0 & 0 & \rho_{4}^{\prime} & 0 & 0 & 0 & 0 & 0 & \rho_{10}^{\prime} & 0 & 0 \\
0 & \rho_{2}^{\prime \prime} & 0 & 0 & 0 & \rho_{6}^{\prime \prime} & 0 & \rho_{8}^{\prime \prime} & 0 & 0 & 0 & \rho_{12}^{\prime \prime} \\
0 & 0 & \rho_{3}^{\prime \prime} & 0 & \rho_{5}^{\prime \prime} & 0 & 0 & 0 & \rho_{9}^{\prime \prime} & 0 & \rho_{11}^{\prime \prime} & 0
\end{array}\right)
$$

$\rho_{j}^{\prime}=d \rho_{j} / d x, \rho_{j}^{\prime \prime}=d^{2} \rho_{j} / d x^{2}$, the mass and stiffness matrices of the beam element can be calculated by formulas

$$
M_{e}=\int_{0}^{L} H^{T}(x)[m] H(x) d x, \quad K_{e}=\int_{0}^{L} \bar{H}^{T}(x)[E] \bar{H}(x) d x .
$$

Index $e$ at $M, K$ shows that the matrices are in local system of co-ordinates.

Thus, the formulation of a beam finite element now leads to the choice of the shape functions $\rho_{j}(x), j=1, \ldots, 12$. In the classical case of beam element, these functions are taken in the form of Hermitian ones:

$$
\begin{aligned}
& \rho_{1}(x)=\rho_{4}(x)=\rho_{1}^{0}(x) \equiv 1-\frac{x}{L} \\
& \rho_{7}(x)=\rho_{10}(x)=\rho_{2}^{0}(x) \equiv \frac{x}{L} \\
& \rho_{2}(x)=-\rho_{3}(x)=\rho_{3}^{0}(x) \equiv 1-3 \frac{x^{2}}{L^{2}}+2 \frac{x^{3}}{L^{3}} \\
& \rho_{5}(x)=\rho_{6}(x)=\rho_{4}^{0}(x)=x\left(1-\frac{x}{L}\right)^{3}, \\
& \rho_{8}(x)=-\rho_{9}(x)=\rho_{5}^{0}(x) \equiv 3 \frac{x^{2}}{L^{2}}-2 \frac{x^{3}}{L^{3}} \\
& \rho_{11}(x)=\rho_{12}(x)=\rho_{6}^{0}(x) \equiv \frac{x^{2}}{L}\left(\frac{x}{L}-1\right) .
\end{aligned}
$$

These functions are the solutions of equations, describing the end conditions

1. $u^{\prime \prime}(x)=0, \quad u(0)=u_{1}, \quad u(L)=u_{2}$,

2. $\theta^{\prime \prime}(x)=0, \quad \theta(x)=\theta_{1}, \quad \theta(L)=\theta_{2}$,

3. $\frac{d^{4} v(x)}{d x^{4}}=0, \quad v(0)=v_{1}, \quad v^{\prime}(0)=v_{1}^{\prime}, \quad v(L)=v_{2}, \quad v^{\prime}(L)=v_{2}^{\prime}$,

4. $\frac{d^{4} w(x)}{d x^{4}}=0 ; w(0)=-w_{1}, w^{\prime}(0)=w_{1}^{\prime}, w(L)=-w_{2}, w^{\prime}(L)=w_{2}^{\prime}$. 
This shows the essence of the finite element approximation. Developing this idea, we will search the shape functions illustrating different cases of damaged beam.

\section{Shape Functions for Damaged Beam Element}

As shown in previous section, the shape-functions are the coefficients at the nodal displacements in the representations of inside beam displacements determined from solving corresponding static problems with given nodal displacements. Basing on this, in present section we will construct the shape functions in two cases of damaged beam: flexible connection and cracked beam elements.

\subsection{Flexible Connection Element}

Let's consider a beam specimen with given parameters: $E, \rho, F, J_{x}, J_{y}, J_{z}$, $L$, connected to the nodes $I, J$ through 8 springs: axial $k_{1}, k_{2}$, torsional $-\nu_{1}^{x}, \nu_{2}^{x}$ and rotational $\nu_{1}^{y}, \nu_{2}^{y}, \nu_{1}^{z}, \nu_{2}^{z}$. Furthermore, the nodal displacements in (2.1) are given, here the problem is to find functions $\rho_{j}, j=1, \ldots, 12$, appeared in (2.2)

a) Axial and Torsion Displacements (Fig. 1)

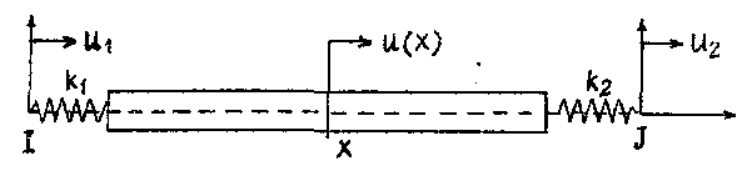

Fig. 1

The axial displacement $u(x)$ of the bar can be found in the form $u(x)=a x+b$ by satisfying conditions:

$$
E F u^{\prime}(0)+k_{1}\left[u(0)-u_{1}\right]=0, \quad E F u^{\prime}(L)+k_{2}\left[u(L)-u_{2}\right]=0 .
$$

Hence, we obtained

$$
a=\frac{u_{1}-u_{2}}{L+\beta_{1}+\beta_{2}}, \quad b=\frac{\beta_{1} u_{2}+\left(L+\beta_{2}\right) u_{1}}{L+\beta_{1}+\beta_{2}}, \quad \beta_{i}=\frac{E F}{k_{i}}, \quad i=1,2 .
$$

In accordance to $(2.2)$, it will be yielded

$$
\begin{aligned}
& \rho_{1}(x)=a_{1}\left(\beta_{1}, \beta_{2}\right)+b_{1}\left(\beta_{1}, \beta_{2}\right) x, \\
& \rho_{7}(x)=a_{2}\left(\beta_{1}, \beta_{2}\right)+b_{2}\left(\beta_{1}, \beta_{2}\right) x,
\end{aligned}
$$

where functions $a_{i}, b_{i}, i=1,2$ of two variables $r_{1}, r_{2}$ are:

$$
\begin{aligned}
& a_{1}\left(r_{1}, r_{2}\right)=\frac{L+r_{2}}{D}, \quad b_{1}\left(r_{1}, r_{2}\right)=-\frac{1}{D} \\
& a_{2}\left(r_{1}, r_{2}\right)=\frac{r_{1}}{D}, \quad b_{2}\left(r_{1}, r_{2}\right)=\frac{1}{D}, \quad D=L+r_{1}+r_{2} .
\end{aligned}
$$


Similarly, for torsion displacement the shape functions $\rho_{4}(x), \rho_{10}(x)$ can be written as

$$
\begin{aligned}
\rho_{4}(x) & =a_{1}\left(\beta_{1 x}, \beta_{2 x}\right)+b_{1}\left(\beta_{1 x}, \beta_{2 x}\right) x, \\
\rho_{10}(x) & =a_{2}\left(\beta_{1 x}, \beta_{2 x}\right)+b_{2}\left(\beta_{1 x}, \beta_{2 x}\right) x,
\end{aligned}
$$

$\beta_{1 x}=G J_{x} / \nu_{1}^{x}, \beta_{2 x}=G J_{x} / \nu_{2}^{x}$ and $a_{i}, b_{i}, i=1,2$ are the same functions as defined in (3.2).

b) Flexural Deflections

For a beam given in Fig. 2 the flexural deflection $v(x)$ can be written as:

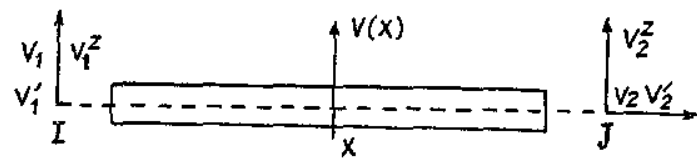

Fig. 2

$$
\begin{gathered}
v(x)=c_{0}+c_{1} x+c_{2} x^{2}+c_{3} x^{3}, \\
w(x)=d_{0}+d_{1} x+d_{2} x^{2}+d_{3} x^{3},
\end{gathered}
$$

which must satisfy conditions:

$$
\begin{aligned}
& v(0)=v_{1}, \quad E J_{z} v^{\prime \prime}(0)-\nu_{1}^{z}\left[v^{\prime}(0)-v_{1}^{\prime}\right]=0, \\
& v(L)=v_{2}, \quad E J_{z} v^{\prime \prime}(L)-\nu_{1}^{z}\left[v^{\prime}(L)-v_{2}^{\prime}\right]=0 \text {, } \\
& w(0)=-w_{1}, \quad E J_{y} w^{\prime \prime}(0)-\nu_{1}^{y}\left[w^{\prime}(0)-w_{1}^{\prime}\right]=0 \text {, } \\
& w(L)=-w_{2}, \quad E J_{y} w^{\prime \prime}(L)-\nu_{1}^{y}\left[w^{\prime}(L)-w_{2}^{\prime}\right]=0 \text {. }
\end{aligned}
$$

The coefficients $c_{i}, d_{i}, i=0,1,2,3$ have been found from the above conditions. Then substituting them into the expressions of $v(x), w(x)$ and comparing with (2.2) we have

$$
\rho_{j}(x)=c_{j 0}+c_{j 1} x+c_{j 2} x^{2}+c_{j 3} x^{3}, \quad j=2,3,5,6,8,9,11,12
$$

with the coefficients

$$
\begin{gathered}
c_{2 i}=a_{1 i}\left(\beta_{1 z}, \beta_{2 z}\right), \quad c_{3 i}=-a_{1 i}\left(\beta_{1 y}, \beta_{2 y}\right), \quad c_{5 i}=a_{2 i}\left(\beta_{1 y}, \beta_{2 y}\right), \\
c_{6 i}=a_{2 i}\left(\beta_{1 z}, \beta_{2 z}\right), \quad c_{8 i}=a_{3 i}\left(\beta_{1 z}, \beta_{2 z}\right), \quad c_{9 i}=-a_{3 i}\left(\beta_{1 y}, \beta_{2 y}\right), \\
c_{11 i}=a_{4 i}\left(\beta_{1 y}, \beta_{2 y}\right) \quad c_{12 i}=a_{4 i}\left(\beta_{1 z}, \beta_{2 z}\right), \quad i=0,1,2,3 \\
\beta_{i y}=\frac{E J_{y}}{\nu_{y}^{i}}, \quad \beta_{i z}=\frac{E J_{z}}{\nu_{z}^{i}}, \quad i=1,2 .
\end{gathered}
$$

functions $a_{j i}\left(r_{1}, r_{2}\right), j=1,2,3,4, i=0,1,2,3$ are given in Table A.1: 


\subsection{Cracked Beam Element}

Consider a beam cracked at a position $\alpha \in(0, L)$. Regarding to changes in geometrical properties of the beam, the crack is assumed to be described by springs: (axial $k$, torsional $\nu_{x}$, bending $\nu_{y}, \nu_{z}$ ), connecting divided beam specimens in both sides of crack position. So, there will be 5 damage parameters $\alpha, \beta=$ $E F / k, \beta_{x}=G J_{x} / \nu_{x}, \beta_{y}=E J_{y} / \nu_{y}, \beta_{z}=E J_{z} / \nu_{z}$ and the shape functions $\rho_{j}(x)$ will be found in the dependence on the damage parameters.

a) Axial and Torsional Model (Fig. 3)
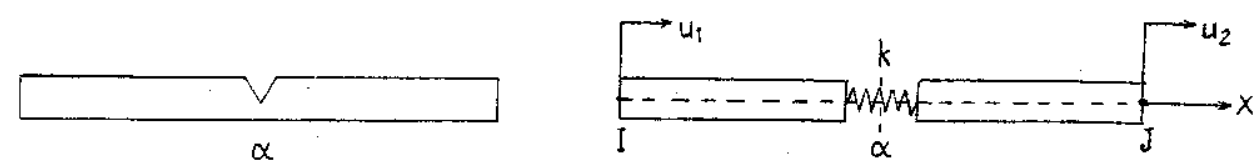

Fig. $\$$

Problem is to find displacement function $u(x)$ of bar at any section $x$ for given nodal displacements $u_{1}, u_{2}$. Such function can be proposed to find in the form

$$
u(x)= \begin{cases}a_{1} x+b_{1} & 0 \leq x<\alpha, \\ a_{2} x+b_{2} & \alpha<x \leq L,\end{cases}
$$

satisfying conditions: $u(0)=u_{1}, u(L)=u_{2}$ and

$$
\begin{aligned}
E F u^{\prime}(\alpha-0)+k[u(\alpha-0)-u(\alpha+0)] & =0 \\
-E F u^{\prime}(\alpha+0)+k[u(\alpha+0)-u(\alpha-0)] & =0
\end{aligned}
$$

The coefficients $a_{i}$ and $b_{i}$ derived from these equations are

$$
a_{1}=\frac{u_{2}-u_{1}}{L+\beta}, \quad b_{1}=u_{1}, \quad a_{2}=\frac{u_{2}-u_{1}}{L+\beta}, \quad b_{2}=\frac{L u_{1}+\beta u_{2}}{L+\beta}, \quad \beta=\frac{E F}{k} .
$$

The function $u(x)$ now has the form,

$$
u(x)= \begin{cases}\rho_{1}^{-} u_{1}+\rho_{7}^{-} u_{2}, & 0 \leq x<\alpha, \\ \rho_{1}^{+} u_{1}+\rho_{7}^{+} u_{2}, & \alpha<x \leq L,\end{cases}
$$

where

$$
\begin{array}{ll}
\rho_{1}^{-}(x)=a_{1}^{-}(\beta)+b_{1}^{-}(\beta) x, & \rho_{7}^{-}(x)=a_{2}^{-}(\beta)+b_{2}^{-}(\beta) x, \\
\rho_{1}^{+}(x)=a_{1}^{+}(\beta)+b_{1}^{+}(\beta)(x-L), & \rho_{7}^{+}(x)=a_{2}^{+}(\beta)+b_{2}^{+}(\beta)(x-L) .
\end{array}
$$

Functions $a_{i}^{ \pm}, b_{i}^{ \pm}, i=1,2$ have the expressions given in Table A.1. 
Similarly, we have

$$
\begin{aligned}
& \rho_{4}^{-}(x)=a_{1}^{-}\left(\beta_{x}\right)+b_{1}^{-}\left(\beta_{x}\right) x, \quad \rho_{10}^{-}(x)=a_{2}^{-}\left(\beta_{x}\right)+b_{2}^{-}\left(\beta_{x}\right) x, \quad \beta_{x}=G J_{x} / \nu_{x}, \\
& \rho_{4}^{+}(x)=a_{1}^{+}\left(\beta_{x}\right)+b_{1}^{+}\left(\beta_{x}\right)(x-L), \quad \rho_{10}^{+}(x)=a_{2}^{+}\left(\beta_{x}\right)+b_{2}^{+}\left(\beta_{x}\right)(x-L),
\end{aligned}
$$

with the same functions $a_{i}^{ \pm}, b_{i}^{ \pm}, i=1,2$, as in (3.6). The found functions do not contain the crack position parameter $\alpha$, they depend only on crack magnitude $\beta$. In addition, if $\beta=0$, then $a_{i}^{+}=a_{i}^{-}, b_{i}^{+}=b_{i}^{-}$or $\rho_{i}^{+}(x)=\rho_{i}^{-}(x)=\rho_{i}^{0}(x)$.

b) Bending Model of Cracked Beam (Fig. 4)

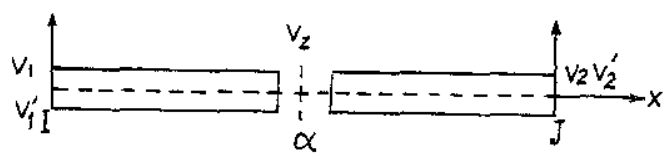

Fig. 4

In this case, the flexural deflections $v(x)$ and $w(x)$ are presented in the forms

$$
\begin{gathered}
v(x)=\left\{\begin{array}{l}
v_{1}+v_{1}^{\prime} x+c_{1} x^{2}+c_{2} x^{3}, \quad 0 \leq x<\alpha, \\
v_{2}+v_{2}^{\prime}(x-L)+c_{3}(x-L)^{2}+c_{4}(x-L)^{4}, \quad \alpha<x \leq L,
\end{array}\right. \\
w(x)=\left\{\begin{array}{l}
-w_{1}+w_{1}^{\prime} x+d_{1} x^{2}+d_{2} x^{3}, \quad 0 \leq x<\alpha, \\
-w_{2}+w_{2}^{\prime}(x-L)+d_{3}(x-L)^{2}+d_{4}(x-L)^{3}, \quad \alpha<x \leq L .
\end{array}\right.
\end{gathered}
$$

It's evident that the functions $v(x), w(x)$ satisfy boundary conditions

$$
\begin{aligned}
v(0) & =v_{1}, \quad v^{\prime}(0)=v_{1}^{\prime}, \quad v(L)=v_{2}, \quad v^{\prime}(L)=v_{2}^{\prime}, \\
w(0) & =-w_{1}, \quad w^{\prime}(0)=w_{1}^{\prime}, \quad w(L)=-w_{2}, \quad w^{\prime}(L)=w_{2}^{\prime} .
\end{aligned}
$$

In order to find $c_{j}, d_{j}, j=1,2,3,4$ there will be following compatibility conditions at crack position $\alpha$ :

$$
\begin{gathered}
v(\alpha-0)=v(\alpha+0), \quad v^{\prime \prime \prime}(\alpha-0)=v^{\prime \prime \prime}(\alpha+0), \\
w(\alpha-0)=\dot{w}(\alpha+0), \quad w^{\prime \prime \prime}(\alpha-0)=w^{\prime \prime \prime}(\alpha+0), \\
E J_{z} v^{\prime \prime}(\alpha-0)+\nu_{z}\left[v^{\prime}(\alpha-0)-v^{\prime}(\alpha+0)\right]=0 \\
E J_{y} w^{\prime \prime}(\alpha-0)+\nu_{y}\left[w^{\prime}(\alpha-0)-w^{\prime}(\alpha+0)\right]=0 \\
E J_{z} v^{\prime \prime}(\alpha+0)-\nu_{z}\left[v^{\prime}(\alpha+0)-v^{\prime}(\alpha-0)\right]=0 \\
E J_{y} w^{\prime \prime}(\alpha+0)+\nu_{y}\left[w^{\prime}(\alpha+0)-w^{\prime}(\alpha-0)\right]=0 .
\end{gathered}
$$

From these equations we can get

$$
\rho_{j}(x)=\left\{\begin{array}{l}
c_{j 0}^{-}+c_{j 1}^{-} x+c_{j 2}^{-} x^{2}+c_{j 3}^{-} x^{3}, 0 \leq x<\alpha, j=2,3,4,5,6,8,9,11,12, \\
c_{j 0}^{+}+c_{j 1}^{+}(x-L)+c_{j 2}^{+}(x-L)^{2}+c_{j 3}^{+}(x-L)^{3}, \alpha<x \leq L
\end{array}\right.
$$


and

$$
\begin{aligned}
c_{2 i}^{ \pm}=a_{1 i}^{ \pm}\left(\alpha_{z}, \beta_{z}\right), & c_{3 i}^{ \pm}=-a_{1 i}^{ \pm}\left(\alpha_{y}, \beta_{y}\right), \quad c_{5 i}^{ \pm}=a_{2 i}^{ \pm}\left(\alpha_{y}, \beta_{y}\right), \\
c_{6 i}^{ \pm}=a_{2 i}^{ \pm}\left(\alpha_{z}, \beta_{z}\right), & c_{8 i}^{ \pm}=a_{3 i}^{ \pm}\left(\alpha_{z}, \beta_{z}\right), \quad c_{9 i}^{ \pm}=-a_{3 i}^{ \pm}\left(\alpha_{y}, \beta_{y}\right), \\
c_{11 i}^{ \pm}=a_{4 i}^{ \pm}\left(\alpha_{y}, \beta_{y}\right), & c_{12 i}^{ \pm}=a_{4 i}^{ \pm}\left(\alpha_{z}, \beta_{z}\right),
\end{aligned}
$$

$i=0,1,2,3$. The functions $a_{i j}^{t}, j=1,2,3,4, i=0,1,2,3$ can be found in Table A.1.

Thus, shape functions for damaged beam elements have been found depending on the damage parameters and all of them are listed in Table 1. It's believed that in any case, if damage magnitudes $\beta$ equal zero the shape functions become to the classical case given by (3.1), which correspond to undamaged beam. Using these shape functions now we can go to the calculation of matrices $M_{e}, K_{e}$.

\section{Calculation of Element Matrices}

By the formulas (2.5) with given form of the matrices $\mathbf{H}, \overrightarrow{\mathbf{H}},[\mathbf{m}],[\mathbf{E}]$, we may write general form of mass and stiffness matrices as

$$
\begin{aligned}
& \mathbf{A}= \\
& {\left[\begin{array}{ccccccccccccc}
a_{11} & & & & & & & & & & & \\
0 & a_{22} & & & & & & & & & & \\
0 & 0 & a_{33} & & & & & & & & \\
0 & 0 & 0 & a_{44} & & & & & & \\
0 & 0 & a_{53} & 0 & a_{55} & & & & & & \\
0 & a_{62} & 0 & 0 & 0 & a_{66} & & & & & & \\
a_{71} & 0 & 0 & 0 & 0 & 0 & a_{77} & & & & & \\
0 & a_{82} & 0 & 0 & 0 & a_{86} & 0 & a_{88} & & & & \\
0 & 0 & a_{93} & 0 & a_{95} & 0 & 0 & 0 & a_{99} & & & \\
0 & 0 & 0 & a_{10,4} & 0 & 0 & 0 & 0 & 0 & a_{10,10} & & \\
0 & 0 & a_{11,3} & 0 & a_{11,5} & 0 & 0 & 0 & a_{11,9} & 0 & a_{11,11} & \\
0 & a_{12,2} & 0 & 0 & 0 & a_{12,6} & 0 & a_{12,8} & 0 & 0 & 0 & a_{12,12}
\end{array}\right]}
\end{aligned}
$$

then

$$
K_{e}=A\left\{a_{i j}=k_{i j}\right\}, \quad M_{e}=\rho F A\left\{a_{i j}=m_{i j}\right\},
$$

where $k_{i j}, m_{i j}$ could be calculated differently regarding to considered beam element's types.

\subsection{Flexible Connection Element}

Using function notations given in Appendix, the elements of mass and stiffness matrices will be found as follows: 


$$
\begin{aligned}
& m_{11}=h_{11}^{f}\left(\beta_{1 u}, \beta_{2 u}\right), \quad m_{71}=h_{12}^{f}\left(\beta_{1 u}, \beta_{2 u}\right), \quad m_{77}=h_{22}^{f}\left(\beta_{1 u}, \beta_{2 u}\right), \\
& m_{44}=h_{11}^{f}\left(\beta_{1 \theta}, \beta_{2 \theta}\right), \quad m_{4,10}=h_{12}^{f}\left(\beta_{1 \theta}, \beta_{2 \theta}\right), \quad m_{10,10}=h_{22}^{f}\left(\beta_{1 \theta}, \beta_{2 \theta}\right) \text {, } \\
& m_{22}=F_{11}^{f}\left(\beta_{1 z}, \beta_{2 z}\right), \quad m_{62}=F_{12}^{f}\left(\beta_{1 z}, \beta_{2 z}\right) ; \quad m_{82}=F_{13}^{f}\left(\beta_{1 z}, \beta_{2 z}\right) \text {, } \\
& m_{33}=F_{11}^{f}\left(\beta_{1 y}, \beta_{2 y}\right), \quad m_{53}=-F_{12}^{f}\left(\beta_{1 y}, \beta_{2 y}\right), \quad m_{12,2}=F_{14}^{f}\left(\beta_{1 z}, \beta_{2 z}\right) \text {, } \\
& m_{93}=F_{13}^{f}\left(\beta_{1 y}, \beta_{2 y}\right), \quad m_{11,3}=-F_{14}^{f}\left(\beta_{1 y}, \beta_{2 y}\right) \quad m_{55}=F_{22}^{f}\left(\beta_{1 y}, \beta_{2 y}\right) \text {, } \\
& m_{95}=-F_{23}^{f}\left(\beta_{1 y}, \beta_{2 y}\right), \quad m_{11,5}=F_{24}^{f}\left(\beta_{1 y}, \beta_{2 y}\right), \quad m_{11,11}=F_{44}^{f}\left(\beta_{1 y}, \beta_{2 y}\right) \text {, } \\
& m_{66}=F_{22}^{f}\left(\beta_{1 z}, \beta_{2 z}\right), \quad m_{86}=F_{23}^{f}\left(\beta_{1 z}, \beta_{2 z}\right), \quad m_{12,6}=F_{24}^{f}\left(\beta_{1 z}, \beta_{2 z}\right) \text {, } \\
& m_{88}=F_{33}^{f}\left(\beta_{1 z}, \beta_{2 z}\right), \quad m_{12.8}=F_{34}^{f}\left(\beta_{1 z}, \beta_{2 z}\right), \quad m_{12,12}=F_{44}^{f}\left(\beta_{1 z}, \beta_{2 z}\right) \text {, } \\
& m_{99}=F_{33}^{f}\left(\beta_{1 z}, \beta_{2 z}\right), \quad m_{11,9}=-F_{34}^{f}\left(\beta_{1 z}, \beta_{2 z}\right) \text {, } \\
& k_{11}=E F g_{11}^{f}\left(\beta_{1 u}, \beta_{24}\right), \quad k_{71}=E F g_{12}^{f}\left(\beta_{1 u}, \beta_{2 u}\right), \quad k_{77}=E F g_{22}^{f}\left(\beta_{1 u}, \beta_{2 u}\right) \\
& k_{44}=G J_{x} g_{11}^{f}\left(\beta_{1 \theta}, \beta_{2 \theta}\right), \quad k_{4,10}=G J_{x} g_{12}^{f}\left(\beta_{1 \theta}, \beta_{2 \theta}\right), \quad k_{10,10}=G J_{x} g_{22}^{f}\left(\beta_{1 \theta}, \beta_{2 \theta}\right) \text {, } \\
& k_{22}=E J_{z} S_{11}^{f}\left(\beta_{1 z}, \beta_{2 z}\right), \quad k_{62}=E J_{z} S_{12}^{f}\left(\beta_{1 z}, \beta_{2 z}\right), \\
& k_{82}=E J_{z} S_{13}^{f}\left(\beta_{1 z}, \beta_{2 z}\right), \quad k_{12,2}=E J_{z} S_{14}^{f}\left(\beta_{1 z}, \beta_{2 z}\right) \text {, } \\
& k_{55}=E J_{y} S_{22}^{f}\left(\beta_{1 y}, \beta_{2 y}\right), \quad k_{95}=-E J_{y} S_{23}^{f}\left(\beta_{1 y}, \beta_{2 y}\right) \text {, } \\
& k_{66}=E J_{z} S_{22}^{f}\left(\beta_{1 z}, \beta_{2 z}\right), \quad k_{11,5}=E J_{y} S_{24}^{f}\left(\beta_{1 y}, \beta_{2 y}\right) \text {, } \\
& k_{86}=E J_{z} S_{23}^{f}\left(\beta_{1 z}, \beta_{2 z}\right), \quad k_{11,11}=E J_{y} S_{44}^{f}\left(\beta_{1 y}, \beta_{2 y}\right) \text {, } \\
& k_{12,6}=E J_{z} S_{24}^{f}\left(\beta_{1 z}, \beta_{2 z}\right), \quad k_{12,12}=E J_{z} S_{44}^{f}\left(\beta_{1 z}, \beta_{2 z}\right) \text {, } \\
& k_{88}=E J_{z} S_{33}^{f}\left(\beta_{1 z}, \beta_{2 z}\right), \quad k_{12,8}=E J_{z} S_{34}^{f}\left(\beta_{1 z}, \beta_{2 z}\right) \text {, } \\
& k_{99}=E J_{z} S_{33}^{f}\left(\beta_{1 z}, \beta_{2 z}\right), \quad k_{11,9}=-E J_{z} S_{34}^{f}\left(\beta_{1 z}, \beta_{2 z}\right) \text {. }
\end{aligned}
$$

\subsection{Cracked Beam Element}

Because of specific form of the shape functions in this case, we will employ the function-notations specified in Appendix, hence, the $M_{e}, K_{e}$ will be calculated as

$$
\begin{aligned}
& m_{11}=h_{11}^{c}\left(\alpha_{u}, \beta_{u}\right), \quad m_{71}=h_{12}^{c}\left(\alpha_{u}, \beta_{u}\right), \quad m_{77}=h_{22}^{c}\left(\alpha_{u}, \beta_{u}\right), \\
& m_{44}=h_{11}^{c}\left(\alpha_{\theta}, \beta_{\theta}\right), \quad m_{4,10}=h_{12}^{c}\left(\alpha_{\theta}, \beta_{\theta}\right), \quad m_{10,10}=h_{22}^{c}\left(\alpha_{\theta}, \beta_{\theta}\right), \\
& m_{22}=F_{11}^{c}\left(\alpha_{z}, \beta_{z}\right), \quad m_{62}=F_{12}^{c}\left(\alpha_{z}, \beta_{z}\right), \quad m_{82}=F_{13}^{c}\left(\alpha_{z}, \beta_{z}\right), \\
& m_{33}=F_{11}^{c}\left(\alpha_{y}, \beta_{y}\right), \quad m_{53}=-F_{12}^{c}\left(\alpha_{y}, \beta_{y}\right), \quad m_{12,2}=F_{14}^{c}\left(\alpha_{z}, \beta_{z}\right), \\
& m_{93}=F_{13}^{c}\left(\alpha_{y}, \beta_{y}\right), \quad m_{11,3}=-F_{14}^{c}\left(\alpha_{y}, \beta_{y}\right), \quad m_{55}=F_{22}^{c}\left(\alpha_{y}, \beta_{y}\right), \ldots \\
& m_{95}=-F_{23}^{c}\left(\alpha_{y}, \beta_{y}\right), \quad m_{11,5}=F_{24}^{c}\left(\alpha_{y}, \beta_{y}\right), \quad m_{11,11}=F_{44}^{c}\left(\alpha_{y}, \beta_{y}\right),
\end{aligned}
$$




$$
\begin{aligned}
& m_{66}=F_{22}^{c}\left(\alpha_{z}, \beta_{z}\right), \quad m_{86}=F_{23}^{c}\left(\alpha_{z}, \beta_{z}\right), \quad m_{12,6}=F_{24}^{c}\left(\alpha_{z}, \beta_{z}\right), \\
& m_{88}=F_{33}^{c}\left(\alpha_{z}, \beta_{z}\right), \quad m_{12,8}=F_{34}^{c}\left(\alpha_{z}, \beta_{z}\right), \quad m_{12,12}=F_{44}^{c}\left(\alpha_{z}, \beta_{z}\right), \\
& m_{99}=F_{33}^{c}\left(\alpha_{z}, \beta_{z}\right), \quad m_{11,9}=-F_{34}^{c}\left(\alpha_{z}, \beta_{z}\right), \\
& k_{11}= E F g_{11}^{c}\left(\alpha_{u}, \beta_{u}\right), \quad k_{71}=E F g_{12}^{c}\left(\alpha_{u}, \beta_{u}\right), \quad k_{77}=E F g_{22}^{c}\left(\alpha_{u}, \beta_{u}\right), \\
& k_{44}= G J_{x} g_{11}^{c}\left(\alpha_{\theta}, \beta_{\theta}\right), \quad k_{4,10}=G J_{x} g_{12}^{c}\left(\alpha_{\theta}, \beta_{\theta}\right), \quad k_{10,10}=G J_{x} g_{22}^{c}\left(\alpha_{\theta}, \beta_{\theta}\right), \\
& k_{22}= E J_{z} S_{11}^{f}\left(\alpha_{z}, \beta_{z}\right), \quad k_{62}=E J_{z} S_{12}^{f}\left(\alpha_{z}, \beta_{z}\right), \quad k_{82}=E J_{z} S_{13}^{f}\left(\alpha_{z}, \beta_{z}\right), \\
& k_{33}=E J_{y} S_{11}^{f}\left(\alpha_{y}, \beta_{y}\right), \quad k_{53}=-E J_{y} S_{12}^{f}\left(\alpha_{y}, \beta_{y}\right), \quad k_{12,2}=E J_{z} S_{14}^{f}\left(\alpha_{z}, \beta_{z}\right), \\
& k_{93}=E J_{y} S_{13}^{f}\left(\alpha_{y}, \beta_{y}\right), \quad k_{11,3}=-E J_{y} S_{14}^{f}\left(\alpha_{y}, \beta_{y}\right), \quad k_{55}=E J_{y} S_{22}^{f}\left(\alpha_{y}, \beta_{y}\right), \\
& k_{95}=-E J_{y} S_{23}^{f}\left(\alpha_{y}, \beta_{y}\right), \quad k_{11,5}=E J_{y} S_{24}^{f}\left(\alpha_{y}, \beta_{y}\right), \quad k_{11,11}=E J_{y} S_{44}^{f}\left(\alpha_{y}, \beta_{y}\right), \\
& k_{66}=E J_{z} S_{22}^{f}\left(\alpha_{z}, \beta_{z}\right), \quad k_{86}=E J_{z} S_{23}^{f}\left(\alpha_{z}, \beta_{z}\right), \quad k_{12,6}=E J_{z} S_{24}^{f}\left(\alpha_{z}, \beta_{z}\right), \\
& k_{88}=E J_{z} S_{33}^{f}\left(\alpha_{z}, \beta_{z}\right), \quad k_{12,8}=E J_{z} S_{34}^{f}\left(\alpha_{z}, \beta_{z}\right), \quad k_{12,12}=E J_{z} S_{44}^{f}\left(\alpha_{z}, \beta_{z}\right), \\
& k_{99}=E J_{z} S_{33}^{f}\left(\alpha_{z}, \beta_{z}\right), \quad k_{11,9}=-E J_{z} S_{34}^{f}\left(\alpha_{z}, \beta_{z}\right) .
\end{aligned}
$$

In formulas (4.2), (4.3) the following notations were introduced: $\alpha_{u}, \alpha_{\theta}, \alpha_{y}, \alpha_{z}$ - positions of crack related to axial, torsion and bending displacements and $\beta_{u}=$ $E F / k, \beta_{\theta}=G J_{x} / \nu_{x}, \beta_{y}=E J_{y} / \nu_{y}, \beta_{z}=E J_{z} / \nu_{z}$ are so-called crack magnitude regarding to mentioned above displacements. This means that the modeled crack related to each displacement may be different, it is easy to verify that crack position does not influence on the axial and torsional behaviour of the element.

\section{Numerical Examples}

\subsection{Flexible Connection}

An example of a beam with two flexural spring at the ends has presented (Fig. 2). The parameters of the beam are as follows: $L=1.0 \mathrm{~m}, E=2.1 \times$ $10^{11} \mathrm{~N} / \mathrm{m}^{2}, F=7.8 \times 10^{-4} \mathrm{~m}^{2}, J=8.4 \times 10^{-8} \mathrm{~m}^{-4}, \rho=7.84 \times 10^{3} \mathrm{~kg} / \mathrm{m}^{3}$. The results illustrated in Fig. 5 present two first frequencies and have received for two cases of $\beta_{1}: 0.0$ (continuous line) and 0.2 (dash line). The parameter $\beta_{2}$ varies on the interval $[0.0,0.9999]$. The lines illustrate the variance of frequencies versus the $\beta_{2}$ (The parameters $\beta_{1}, \beta_{2}$ have the same role in variance of the frequencies). It can be seen from the results, that for different values $\beta_{1}$ the lines are shifting down from the line $\beta_{1}=0.0$. The presented result is similar to that obtained in [7]. A difference here is that there is taken into consideration the spring flexibility instead of its stiffness.

\subsection{Cracked Beam Element}

In another example, a fixed-end beam with a flexural spring describing a crack 
at a position $x$ is investigated. The parameters of the beam are as the same as the beam described in the example 1. The frequencies, which are illustrated in Fig. 6 , have been computed for four different values of $\beta=0.0,0.1,1.0,5.0$. The graphics presented in Fig. 6 give the relationship between frequencies and the position of crack. The results show good agreement with theoretical ones given in [14]. An interest could be seen from the obtained result is that there are positions along the beam at which crack does not influence on the frequencies. This fact is useful for detecting crack position from measured natural frequencies.
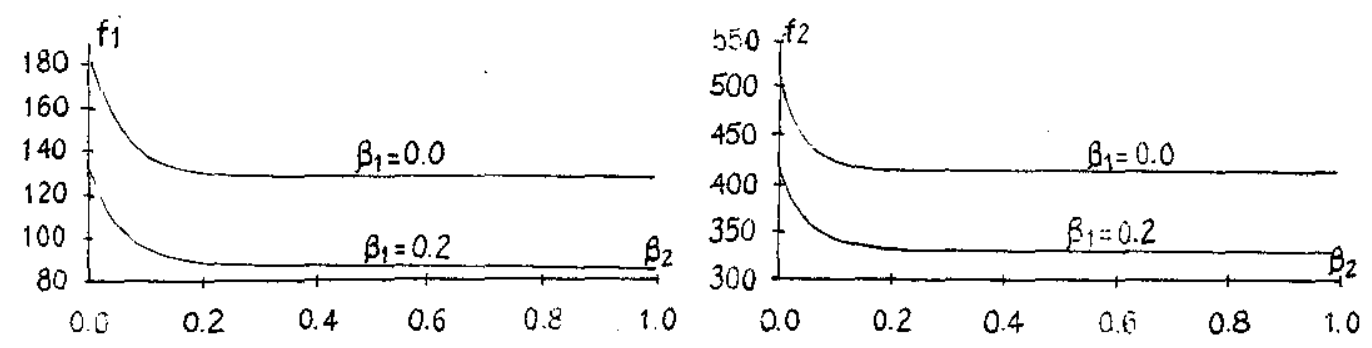

Fig. 5
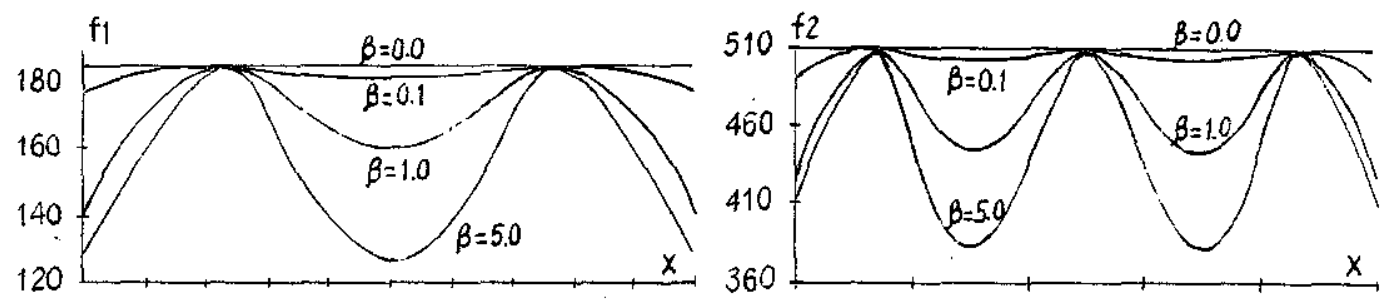

Fig. 6

\subsection{Damaged frame structure}

There will be considered the steel frame that was investigated in [7], see Fig. 7 . Thus, there is a plan frame structure (with height $7.3 \mathrm{~m}$, wide $6.1 \mathrm{~m}$ ) consisting of 6 nodes and 6 circular beam $(\phi=0.1 \mathrm{~m})$ elements. Flexible connections of different relative flexibility values will be introduced at the nodes connecting the columns with the base and the horizontal braces with columns. Cracks are assumed in the braces and columns at various positions. The flexible connection and crack are investigated only in their bending model. The subject of this calculation is change in the natural frequencies of the structure. The cases of damage proposed for consideration are as follows:

Case A: Flexible connection of the same rotation flexibility $(\beta)$ is introduced simultaneously at the nodes connecting columns with base.

Case B: Flexible connection at both ends of the middle horizontal brace (MHB) with columns. 
Case $C$ : Flexible connection at both ends of the top horizontal brace (THB) with columns.

Case D: Crack appeared in the MHB at various positions $(\alpha)$ and with different values of magnitude $(\beta)$.

Case $E$ : Crack appeared in the THB at various positions $(\alpha)$ and with different values of magnitude $(\beta)$.

Case $F$ : Crack appeared in only one of the columns at various positions $(\alpha)$ and with different values of magnitude $(\beta)$.

Case $G$ : Crack appeared in the both columns at various positions $\left(\alpha_{1}, \alpha_{2}\right)$ and with different values of magnitude $\left(\beta_{1}, \beta_{2}\right)$.

Results of computation, the first two natural frequencies corresponding to the specified above cases are given in Table 1.

Analysis of the results leads to the following conclusions:

1. Damage, either flexible connection or crack in the bending model does not changes the third natural frequency of the frame.

2. Reduction of the stiffness at the joint nodes (flexible connection):

- Weakening of connection of the structure with base can decrease maximum the first frequency $40 \%$ and the second one $18 \%$. Decrease of the both frequencies versus weakness of the connection stiffness is almost monotonous.

- Flexible connection of the MHB with columns does not influences on the second frequency, while change of the connection between THB and columns leads to decrease of the both frequencies.

- The more distance of the flexible connection from the base is, the less its infiuence on the natural frequencies.

3. Appearance of crack:

- Crack appeared at middle of the horizontal elements with any magnitude does not changes the second frequency. Influence of the cracks appeared symmetrically in both sides of the middle is the same and this influence increases as the crack goes far-away from the middle.

- If crack appeared in a column, the more closed to the base crack position is, the more decrease of the first frequency and the less change in the second one. In this case change in frequencies is not symmetrical as in the horizontal members, minimal change of frequencies is recognised at the upper half of member.

- Simultaneously appeared at both columns cracks make more change in the frequencies than that if crack is only in one column. Unequal of the distances of the cracks in columns from the base inflects also to the change of frequencies. This change is always less than that if cracks appeared at the least of distance from base.

- In general, the more magnitude of crack, the more decrease of frequencies. 
Table 1

\begin{tabular}{|c|c|c|c|c|c|c|c|c|c|c|c|}
\hline Case & $\beta$ & 0.0 & 0.1 & -0.2 & 0.4 & 0.6 & 0.8 & 1.0 & 2.0 & 4.0 & $\infty$ \\
\hline \multirow[t]{2}{*}{ A } & 1 freq. & 1.76 & 1.69 & 1.62 & 1.51 & 1.42 & 1.36 & 1.30 & 1.15 & 1.06 & 1.00 \\
\hline & 2 freq. & 5.60 & 5.44 & 5.31 & 5.13 & 5.01 & 4.93 & 4.87 & 4.74 & 4.67 & 4.64 \\
\hline \multirow[t]{2}{*}{ B } & 1 freq. & 1.76 & 1.71 & 1.66 & 1.58 & 1.52 & 1.47 & 1.44 & 1.34 & 1.27 & - \\
\hline & 2 freq. & 5.60 & 5.60 & 5.60 & 5.60 & $5.60^{\circ}$ & 5.60 & 5.60 & 5.60 & 5.60 & - \\
\hline \multirow[t]{4}{*}{$\mathrm{C}$} & 1 freq. & 1.76 & 1.74 & 1.72 & 1.67 & 1.63 & 1.60 & 1.57 & 1.47 & 1.39 & - \\
\hline & 2 freq. & 5.60 & 5.50 & 5.42 & 5.26 & 5.24 & 5.05 & 4.97 & 4.75 & 4.61 & - \\
\hline & $\beta$ & & & 0.1 & & & & 1.0 & & 2. & 0 \\
\hline & $\alpha$ & $\mathrm{L} / 8$ & $\mathrm{~L} / 4$ & $\mathrm{~L} / 2$ & $3 \mathrm{~L} / 4$ & $7 \mathrm{~L} / 8$ & $\mathrm{~L} / 8$ & $\mathrm{~L} / 4$ & $\mathrm{~L} / 2$ & $\mathrm{~L} / 8$ & $\mathrm{~L} / 4$ \\
\hline \multirow[t]{2}{*}{$\mathrm{D}$} & 1 freq. & 1.68 & 1.72 & 1.76 & 1.72 & 1.68 & 1.51 & 1.62 & 1.76 & 1.49 & 1.60 \\
\hline & 2 freq. & 5.60 & 5.60 & 5.60 & 5.60 & 5.60 & 5.60 & 5.60 & 5.60 & 5.60 & 5.60 \\
\hline \multirow[t]{4}{*}{$\mathrm{E}$} & 1 freq. & 1.73 & 1.75 & 1.76 & 1.75 & 1.73 & 1.62 & 1.69 & 1.76 & 1.61 & 1.68 \\
\hline & 2 freq. & 5.45 & 5.53 & 5.60 & 5.53 & 5.45 & 5.11 & 5.32 & 5.60 & 5.07 & 5.28 \\
\hline & $\beta$ & & & 0.1 & & & & & 1.0 & & \\
\hline & $\alpha$ & $\mathrm{L} / 8$ & $\mathrm{~L} / 4$ & $\mathrm{~L} / 2$ & $3 \mathrm{~L} / 4$ & $7 \mathrm{~L} / 8$ & $\mathrm{~L} / 8$ & $\mathrm{~L} / 4$ & $\mathrm{~L} / 2$ & $3 \mathrm{~L} / 4$ & $7 \mathrm{~L} / 8$ \\
\hline \multirow[t]{5}{*}{$\mathrm{F}$} & 1 freq. & 1.69 & 1.72 & 1.76 & 1.76 & 1.75 & 1.56 & 1.61 & 1.73 & 1.76 & 1.71 \\
\hline & 2 freq. & 5.47 & 5.54 & 5.60 & 5.52 & 5.43 & 5.25 & 5.40 & 5.60 & 5.24 & 4.99 \\
\hline & \multicolumn{11}{|c|}{$\beta_{1}=\beta_{2}=2.0$} \\
\hline & $\alpha_{1}$ & & & $\mathrm{~L} / 8$ & & & & $\mathrm{~L}$ & $/ 2$ & & $3 \mathrm{~L} / 4$ \\
\hline & $\alpha_{2}$ & $\mathrm{~L} / 8$ & $\mathrm{~L} / 4$ & $\mathrm{~L} / 2$ & $3 \mathrm{~L} / 4$ & $7 \mathrm{~L} / 8$ & $\mathrm{~L} / 8$ & $\mathrm{~L} / 4$ & $\mathrm{~L} / 2$ & $7 \mathrm{~L} / 8$ & $\mathrm{~L} / 8$ \\
\hline \multirow[t]{2}{*}{$\mathrm{G}$} & 1 freq. & 1.18 & 1.28 & 1.49 & 1.50 & 1.41 & 1.49 & 1.55 & 1.68 & 1.66 & 1.50 \\
\hline & 2 freq. & 4.87 & 5.01 & 5.23 & 4.81 & 4.56 & 5.23 & 5.38 & 5.60 & 4.93 & 4.81 \\
\hline
\end{tabular}

\section{General Conclusion}

Two cases of modified the beam finite element were developed to study frame structures with flexible connections and cracked members. The flexible connection and crack have been modeled by different types of spring, relative flexibility of which together with crack position serve as damage parameters. These ones 
take part into new form of the shape functions, consequently, the consistent mass and stiffness matrices of the structure were constructed also as functions of the damage parameters. Thus, by the same procedure of FEM there can be analyzed behaviour of the frame structures in dependence on the appearance of damage. The developed beam elements have been compiled with SAP IV and made the SAP to get a new development, which can be employed to analysis of damaged frame structures. Presented here numerical examples show the efficiency and applicable of the development. The general result obtained in this study were applied to analysis and integrity assessment of the oil production offshore platforms operating in Bach Ho field, Vietnam.

This study was completed by financial supports from The National Program for Natural scientific research and The Research Program of National Centre for Natural Sciences and Technology of Vietnam.
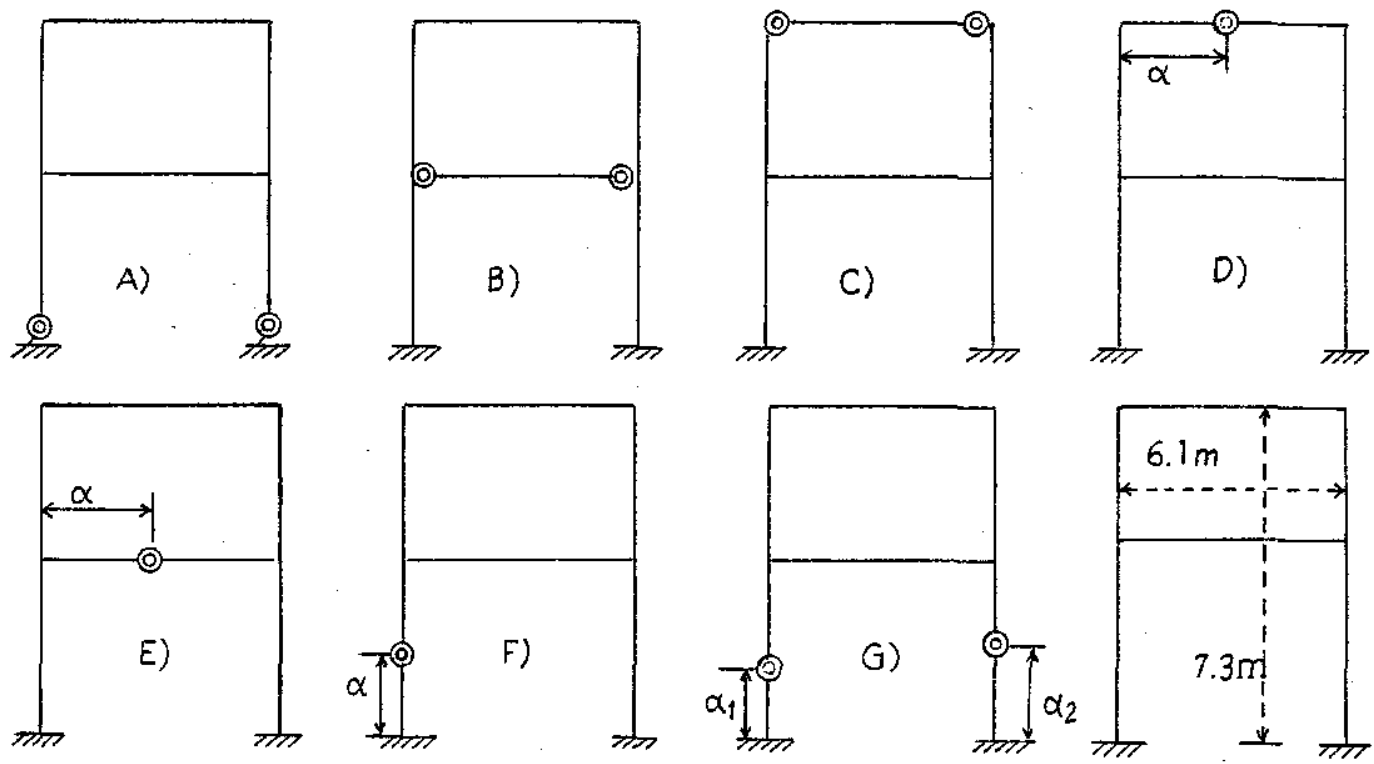

Fig. 7

APPENDIX- FUNCTION NOTATIONS

a. For flexible connection element

$$
\begin{aligned}
h_{i j}^{f}\left(r_{1}, r_{2}\right) & =\int_{0}^{L}\left[a_{i}\left(r_{1}, r_{2}\right)+x b_{i}\left(r_{1}, r_{2}\right)\right]\left[a_{j}\left(r_{1}, r_{2}\right)+x b_{j}\left(r_{1}, r_{2}\right)\right] d x \\
& =L a_{i} a_{j}+\frac{1}{2} L^{2}\left(a_{i} b_{j}+a_{j} b_{i}\right)+\frac{1}{3} L^{3} b_{i} b_{j}, \quad i, j=1,2
\end{aligned}
$$




$$
\begin{gathered}
F_{i j}^{f}\left(r_{1} \cdot r_{2}\right)=\int_{0}^{L} \sum_{k_{1}, k_{2}}^{3} a_{i k_{1}} a_{j k_{2}} x^{k_{1}} x^{k_{2}} d x=\sum_{k=1}^{7} b_{i j}\left(k, r_{1}, r_{2}\right) L^{k} \\
b_{i j}=\frac{1}{k} \sum_{p=0}^{k-1} a_{i \ell} a_{j, k-\ell-p}, \quad i, j=1,2,3,4, \\
g_{i j}^{f}\left(r_{1}, r_{2}\right)=b_{i}\left(r_{1}, r_{2}\right) b_{j}\left(r_{1}, r_{2}\right) L, \quad i, j=1,2, \\
\left.S_{i j}^{f}\left(r_{1}, r_{2}\right)=\int_{0}^{L}\left[2 a_{i 2}\left(r_{1}, r_{2}\right)+6 a_{i 3} r_{1}, r_{2}\right) x\right]\left[2 a_{j 2}\left(r_{1}, r_{2}\right)+6 a_{j 3}\left(r_{1}, r_{2}\right) x\right] d x \\
=4 L a_{i 2} a_{j 2}+6 L^{2}\left(a_{i 2} a_{j 3}+a_{i 3} a_{j 2}\right)+12 L^{3} a_{i 3} a_{j 3}, \quad i, j=1,2,3,4 .
\end{gathered}
$$

b. For cracked element

$$
\begin{aligned}
& h_{i j}^{+}\left(r_{1}, r_{2}\right)=a_{i}^{+} a_{j}^{+}\left(r_{1}-L\right)+\frac{1}{2}\left[a_{i}^{+} b_{j}^{+}+a_{j}^{+} b_{i}^{+}\right]\left(r_{1}-L\right)^{2}+\frac{1}{3} b_{i}^{+} b_{j}^{+}\left(r_{1}-L\right)^{3}, \\
& a_{i}^{ \pm}=a_{i}(r), \quad b_{i}^{ \pm}=b_{i}(r), \\
& h_{i j}^{-}\left(r_{1}, r_{2}\right)=a_{i}^{-} a_{j}^{-} r_{1}+\frac{1}{2}\left[a_{i}^{-} b_{j}^{-}+a_{j}^{-} b_{i}^{-}\right] r_{1}^{2}+\frac{1}{3} b_{i}^{-} b_{j}^{-} r_{1}^{3} \text {, } \\
& h_{i j}^{c}(r)=h_{i j}^{-}(r)-h_{i j}^{+}(r) \text {, } \\
& F_{i j}^{ \pm}\left(r_{1}, r_{2}\right)= \\
& = \begin{cases}\sum_{k=1}^{7} b_{i j k}^{+}\left(r_{1}, r_{2}\right) r_{1}^{k}, & b_{i j k}^{+}=\frac{1}{k} \sum_{p=0}^{k-1} a_{i p}^{+} a_{j, k-\ell-p}^{+}, a_{i j}^{ \pm}=a_{i j}^{ \pm}\left(r_{1}, r_{2}\right) \\
\sum_{k=1}^{7} b_{i j k}^{-}\left(r_{1}, r_{2}\right)\left(r_{1}-L\right)^{k}, & b_{i j k}^{-}=\frac{1}{k} \sum_{p=0}^{k-1} a_{i p}^{-} a_{j, k-\ell-p}^{-}, i, j=1,2,3,4\end{cases} \\
& F_{i j}^{c}\left(r_{1}, r_{2}\right)=F_{i j}^{-}\left(r_{1}, r_{2}\right)-F_{i j}^{+}\left(r_{1}, r_{2}\right) \text {, } \\
& g_{i j}^{ \pm}\left(r_{1}, r_{2}\right)=\left\{\begin{array}{l}
b_{i}^{+}\left(r_{2}\right) b_{j}^{+}\left(r_{2}\right)\left(r_{1}-L\right) \\
b_{i}^{-}\left(r_{2}\right) b_{j}^{-}\left(r_{2}\right) r_{1}
\end{array}, i, j=1,2\right. \\
& g_{i j}^{c}\left(r_{1}, r_{2}\right)=g_{i j}^{-}\left(r_{1}, r_{2}\right)-g_{i j}^{+}\left(r_{1}, r_{2}\right), \\
& S_{i j}^{+}\left(r_{1}, r_{2}\right)=4 a_{i 2}^{+} a_{j 2}^{+}\left(r_{1}-L\right)+6\left(a_{i 2}^{+} a_{j 3}^{+}+a_{i 3}^{+} a_{j 2}^{+}\right)\left(r_{1}-L\right)^{2}+12 a_{i 3}^{+} a_{j 3}^{+}\left(r_{1}-L\right)^{3} \\
& S_{i j}^{-}\left(r_{1}, r_{2}\right)=4 a_{i 2}^{-} a_{j 2}^{-} r_{1}+6\left(a_{i 2}^{-} a_{j 3}^{-}+a_{i 3}^{-} a_{j 2}^{-}\right) r_{1}^{2}+12 a_{i 3}^{-} a_{j 3}^{-} r_{1}^{3}, \quad i, j=1,2,3,4 \\
& S_{i j}^{\mathrm{c}}\left(r_{1}, r_{2}\right)=S_{i j}^{-}\left(r_{1}, r_{2}\right)-S_{i j}^{+}\left(r_{1}, r_{2}\right)
\end{aligned}
$$


Table A.1. Coefficients of Shape Functions

\begin{tabular}{|c|c|c|c|c|c|c|}
\hline \multirow{2}{*}{\multicolumn{2}{|c|}{ Classic }} & \multirow{3}{*}{$\frac{\begin{array}{c}\text { Flexible } \\
\text { connection }\end{array}}{1}$} & \multicolumn{4}{|c|}{ Cracked } \\
\hline & & & \multicolumn{2}{|r|}{$0 \leq x \leq r_{1}$} & \multicolumn{2}{|r|}{$r_{1}<x \leq L$} \\
\hline$a_{10}$ & 1 & & $a_{10}^{-}$ & 1. & $a_{10}^{+}$ & 0 \\
\hline$a_{11}$ & 0 & $\frac{6 r_{1}\left(L+2 r_{2}\right)}{D_{f} L}$ & $a_{11}^{\overline{1}}$ & 0 & $a_{11}^{+}$ & 0 \\
\hline$a_{12}$ & $\frac{-3}{L^{2}}$ & $\frac{-3\left(L+2 r_{2}\right)}{D_{f} L}$ & $\overrightarrow{a_{12}}$ & $\frac{-3\left(L^{2}+2 r_{1} r_{2}\right)}{D_{c}}$ & $a_{12}^{+}$ & $\frac{3\left[L^{2}-2 r_{2}\left(r_{1}-L\right)\right]}{D_{c}}$ \\
\hline$a_{13}$ & $\frac{2}{L^{3}}$ & $\frac{2 d_{f}}{D_{f} L^{2}}$ & $a_{13}^{-}$ & $\frac{2\left(L+r_{2}\right)}{D_{c}}$ & $a_{13}^{+}$ & $\frac{2\left(L+r_{2}\right)}{D_{c}}$ \\
\hline$a_{20}$ & 0 & 0 & $\overrightarrow{a_{20}}$ & 0 & $a_{20}^{+}$ & 0 \\
\hline$a_{21}$ & 1 & $\frac{L\left(L+4 r_{2}\right)}{\bar{D}_{f}}$ & $a_{21}^{-}$ & 1 & $a_{21}^{+}$ & 0 \\
\hline$a_{22}$ & $\frac{-2}{L}$ & $\frac{-2\left(L+3 r_{2}\right)}{D_{f}}$ & $a_{22}^{-}$ & $\frac{-2\left(L^{3}+3 r_{1}^{2} r_{2}\right)}{D_{c}}$ & $a_{22}^{+}$ & $\frac{L^{3}-6 r_{1} r_{2}\left(r_{1}-L\right)}{D_{c}}$ \\
\hline$a_{23}$ & $\frac{1}{L^{2}}$ & $\frac{L+2 r_{2}}{D_{f} L}$ & $a_{23}^{-}$ & $\frac{L^{2}+2 r_{1} r_{2}}{D_{c}}$ & $a_{23}^{+}$ & $\frac{L^{2}+2 r_{1} r_{2}}{D_{c}}$ \\
\hline$a_{30}$ & 0 & 0 & $a_{30}^{-}$ & 0 & $a_{30}^{+}$ & 1. \\
\hline$a_{31}$ & 0 & $\frac{6 r_{1}\left(L+2 e_{2}\right)}{D_{f} L}$ & $a_{31}^{-}$ & 0 & $a_{31}^{+}$ & 0 \\
\hline$a_{32}$ & $\frac{3}{L^{2}}$ & $\frac{3\left(L+2 r_{2}\right)}{D_{f} L}$ & $a_{32}^{-}$ & 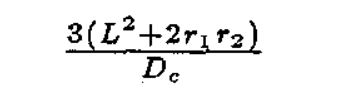 & $a_{32}^{+}$ & $\frac{-3\left[L^{2}-2 r_{2}\left(r_{1}-L\right)\right]}{D_{c}}$ \\
\hline$a_{33}$ & $\frac{-2}{L^{3}}$ & $\frac{-2 d_{f}}{D_{f} L^{2}}$ & $a_{33}^{-}$ & $\frac{-2\left(L+r_{2}\right)}{D_{c}}$ & $a_{33}^{+}$ & $\frac{-2\left(L+r_{2}\right)}{D_{c}}$ \\
\hline$a_{40}$ & 0 & 0 & $a_{40}^{-}$ & 0 & $a_{40}^{+}$ & 0 \\
\hline$a_{41}$ & 0 & $\frac{-2 L_{r_{1}}}{\bar{D}_{f}}$ & $a_{41}$ & 0 & $a_{41}^{+}$ & 1 \\
\hline$a_{42}$ & $\frac{-1}{L}$ & $\frac{-L}{D_{f}}$ & $a_{42}^{-}$ & $\frac{-\left\{L^{3}-6 r_{1} r_{2}\left(r_{1}-L\right)\right]}{D_{c}}$ & $a_{42}^{+}$ & $\frac{2\left[L^{3}+3 r_{2}\left(r_{1}-L\right)^{2}\right]}{D_{0}}$ \\
\hline$a_{43}$ & $\frac{1}{L^{2}}$ & $\frac{L+2 r_{2}}{D_{f}}$ & $a_{43}^{-}$ & $\frac{2\left(L+r_{2}\right)}{D_{c}}$ & $a_{43}^{+}$ & $\frac{L-2 r_{2}\left(r_{1}-L\right)}{D_{c}}$ \\
\hline
\end{tabular}

$$
\begin{gathered}
d_{f}=L+r_{1}+r_{2} \\
D_{f}=L^{2}+4 L\left(r_{1}+r_{2}\right)+12 r_{1} r_{2}
\end{gathered}
$$




$$
\begin{gathered}
d_{c}=L+r_{2} \\
D_{c}=L\left(L^{3}+12 r_{1} r_{2}\left(r_{1}-L\right)+4 L^{2} r_{2}\right) \\
\rho_{1}=a_{1}+b_{1} x, \quad \rho_{2}=a_{2}+b_{2} x \\
\rho_{j}=C_{j 0}+C_{j 1} x+C_{j 1} x^{2}+C_{j 1} x^{3}, \quad j=3,4,5,6 \\
\rho_{i}=a_{i}^{-}+b_{i}^{-} x, 0 \leq x<r_{1}, \rho_{i}=a_{i}^{+}+b_{i}^{+}(x-L), r_{1} \leq x<L, i=1,2 \\
\rho_{j}=a_{j 0}^{-}+a_{j 1}^{-} x+a_{j 1}^{-} x^{2}+a_{j 2}^{-} x^{3}, \quad 0 \leq x<r_{1}, \\
\rho_{j}=a_{j 0}^{+}+a_{j 1}^{+}(x-L)+a_{j 2}^{+}(x-L)^{2}+a_{j 3}^{+}(x-L)^{3}, \quad r_{1} \leq x<L \\
j=3,4,5,6
\end{gathered}
$$

\section{References}

1. Bathe K. J. Finite Element Procedure in Engineering Analysis, Prentice-Hall, NJ, 1982.

2. Lui E. M. and Chen W. F. "Steel Frame Analysis with Flexible Joints", J. Construct. Steel Research, 8 1987, pp. 162-202.

3. Lui E .M. and Chen W. F. "Behaviour of Braced and Un-Braced Semi-Rigid Frame", Int. J. Solids Structures, 24 (9) 1988, pp. 843-931.

4. Carlo Poggi, "A Finite Element Model for Analysis of Flexible Connected Steel Frames", Int. J. Num. Methods Eng., 26 1988, pp. 2239-2254.

5. Shi G. and Atluri S. N. "Static and Dynamic-Analysis of Space Frames with Non-linear Flexible Connection", Int. J. Num. Methods. Eng., 28 1989, pp. 2635-2650.

6. Kawashima S. and Fujimoto T. "Vibration Analysis of Frame with Semi-Rigid connections", Comp. Struct., 19 1984, pp. 85-92.

7. Chan S. L. and Ho W. M. "Non-linear Vibration Analysis of Steel Frames with Semi-rigid Connections", J. Structural Engineering, 120 (4) 1994, pp. 1075-1087.

8. Pedersen P. "A Note on Vibration of Beam-Columns", J. Sound and Vibration, 105 (1) 1986, pp. 143-150.

9. Gudmundson P. "The Dynamic Behaviour of Slender Structures with CrossSectional Cracks", J. Mech. and Phys. Solids, 31 (4) 1983, pp. 329-345.

10. Christides S. and Barr A. D. S. "One-Dimensional Theory of Cracked Bernoulli-Euler Beams", Int. J. Mech. Sciences, 26 (11/12)m 1984, pp. 115-134.

11. Shen M. H. H. and Pierre C. "Natural Mode of Bernoulli-Euler Beams with Symmetric Cracks", J. Sound and Vibration, 1938 (1) 1990, pp. 115-134. 
12. Pizos P. E., Aspragathos N. and Dimarogonas A. D. "Identification of Crack Location and Magnitude in a Cantilever Beam from the Vibration Modes", J. Sound and Vibration, 138 (3) 1990, pp. 381-388,

13. Nakis Y. "Identification of Crack Location in Vibrating Simply Supported Beams". J. Sound and Vibration, 172 (4) 1994, pp. 549-559.

14. Nguyen Tien Khiem, Dao Nhu Mai. "Natural frequency analysis of cracked beam". J. Mechanics (to be appeared).

Received Appril 12, 1998

\section{PHÂN TÍCH ĐộNG KẾT CẤU KHÔNG NGUYÊN VẸN BĂNG PHUƠNG PHÁP PHẦ TỬ HŨU HAN CẢI BIÊN}

Phần tử dầm cổ điển đã được cải biên và phát triển để phân tích dao động của một khung không gian với các liên kết mềm và các phần tứ có vết nứt. $\mathrm{Ma}$ trận độ cứng và ma trận khối lượng được thiết lập cho các phần tứ dầm cải biên này dựa trên hàm dạng mới, được xây dựng qua việc mô hình liên kết mềm và vết nứt bằng các lò xo tương đương. Hàm dạng này vẫn có dạng đa thức bậc ba và có chứa các tham số của liên kết (của vết nứt). Chúng không làm thay đồi quy trình thường dùng của việc mô hình hóa bằng phương pháp PTHH. Do vậy, phương pháp đưa ra ở đây dề dàng áp dựng để phân tích tham số trong bài toán trị riêng của các kết cấu theo các tham số của liên kết mềm (hay vết nứt). Cách tiếp cận này đã được áp dụng để tính các giá trị riêng và dạng riêng của kết cấu khung cho một số ví dụ. 